\title{
Experimental Observation of Rainbow Scattering by a Coated Cylinder: Twin Primary Rainbows and Thin-Film Interference
}

Charles L. Adler

James A. Lock

Cleveland State University, j.lock@csuohio.edu

Justin K. Nash

Follow this and additional works at: https://engagedscholarship.csuohio.edu/sciphysics_facpub Kirk W. Saunders

Part of the Physics Commons

How does access to this work benefit you? Let us know!

\section{Publisher's Statement}

This paper was published in Applied Optics and is made available as an electronic reprint with the permission of OSA. The paper can be found at the following URL on the OSA website: http://www.opticsinfobase.org/ao/abstract.cfm?URI=ao-40-9-1548. Systematic or multiple reproduction or distribution to multiple locations via electronic or other means is prohibited and is subject to penalties under law.

\section{Original Citation}

Adler, Charles L., James A. Lock, Justin K. Nash, and Kirk W. Saunders. "Experimental Observation of Rainbow Scattering by a Coated Cylinder: Twin Primary Rainbows and Thin-Film Interference." Applied Optics 40 (2001): 1548-1558.

\section{Repository Citation}

Adler, Charles L.; Lock, James A.; Nash, Justin K.; and Saunders, Kirk W., "Experimental Observation of Rainbow Scattering by a Coated Cylinder: Twin Primary Rainbows and Thin-Film Interference" (2001). Physics Faculty Publications. 77.

https://engagedscholarship.csuohio.edu/sciphysics_facpub/77

This Article is brought to you for free and open access by the Physics Department at EngagedScholarship@CSU. It has been accepted for inclusion in Physics Faculty Publications by an authorized administrator of EngagedScholarship@CSU. For more information, please contact library.es@csuohio.edu. 


\title{
Experimental observation of rainbow scattering by a coated cylinder: twin primary rainbows and thin-film interference
}

\author{
Charles L. Adler, James A. Lock, Justin K. Nash, and Kirk W. Saunders
}

\begin{abstract}
We experimentally examine the primary rainbow created by the illumination of a coated cylinder. We present a simple technique for varying the coating thickness over a wide range of values, and we see evidence for two different scattering regimes. In one, where the coating thickness is large, twin rainbows are produced. In the second, where the coating is thin enough to act as a thin film, a single rainbow is produced whose intensity varies periodically as the coating thickness varies. We find good agreement with previous theoretical predictions. (C) 2001 Optical Society of America
\end{abstract}

OCIS codes: $290.0290,290.4020,120.0120,120.4120$.

\section{Introduction}

Since the early 1900's there has been a great deal of interest in rainbow scattering by nonspherical or inhomogeneous particles. ${ }^{1-4}$ This interest was greatest in the mid-1970's to the early 1980's, when catastrophe theory revolutionized our understanding of the rainbow caustic, ${ }^{5-12}$ and again in the mid1990 's to the present, as rainbow refractometry became a practical tool for particle sizing and index measurement. ${ }^{13-25}$ In the latter studies, index inhomogeneity is of great concern because it will lead to errors in the measurement of droplet temperature and index of refraction. Although there have been a number of studies on the effects of index inhomogeneities on the rainbow position by use of sophisticated multilayer models of liquid droplets, to our knowledge there have been no experimental studies of such systems. ${ }^{26-32}$ This is due to the experimental difficulty in creating a droplet in which the index of refraction can be varied spatially in a controllable way.

In this paper we study the simplest possible case of index inhomogeneity, namely, rainbow scattering by

C. L. Adler (cladler@osprey.smcm.edu), J. K. Nash, and K. W. Saunders are with the Department of Physics, St. Mary's College of Maryland, St. Mary's City, Maryland 20686. J. A. Lock is with the Department of Physics, Cleveland State University, Cleveland, Ohio 44115.

Received 10 August 2000; revised manuscript received 5 December 2000 .

0003-6935/01/091548-11\$15.00/0

(C) 2001 Optical Society of America a coated particle - that is, one that can be divided into two regions: the core with index of refraction $n_{1}$ and the coating, which completely surrounds the core, with index $n_{2}$; see Fig. 1 . (We assume that the medium surrounding the particle has an index of refraction of 1.0.) The theory of rainbow scattering has already been carried out for a special class of coated particle, namely, the coated sphere. ${ }^{33}$ In that paper, rainbow scattering by a coated sphere was investigated with geometrical optics and extensions to Mie theory. Although we do not examine the coated sphere in this paper, most of the results of Ref. 33 can be adopted by us with no alteration for the class of particles we are studying: coated cylinders illuminated normally to the cylinder axis. Rainbow scattering by two concentric cylinders with circular cross sections is identical (as far as geometrical optics is concerned) to scattering in an equatorial plane of a coated sphere whose core and coating radii and indices are identical to those of the cylinder.

Even though this system is simple, there is interesting physics here. Several specific predictions have been made for rainbow scattering by singly coated particles. As the coating thickness varies, the primary rainbow should vanish for large coating thicknesses, be twinned (i.e., two rainbows emergent in place of one) for intermediate thicknesses (referred to as the $\alpha$ and $\beta$ rainbows), or vary in brightness periodically when the coating is only a few wavelengths thick. ${ }^{33}$ In this paper we report the observation of the latter two phenomena. In addition, we also studied a doubly coated cylinder and have seen several phenomena that have never been predicted 


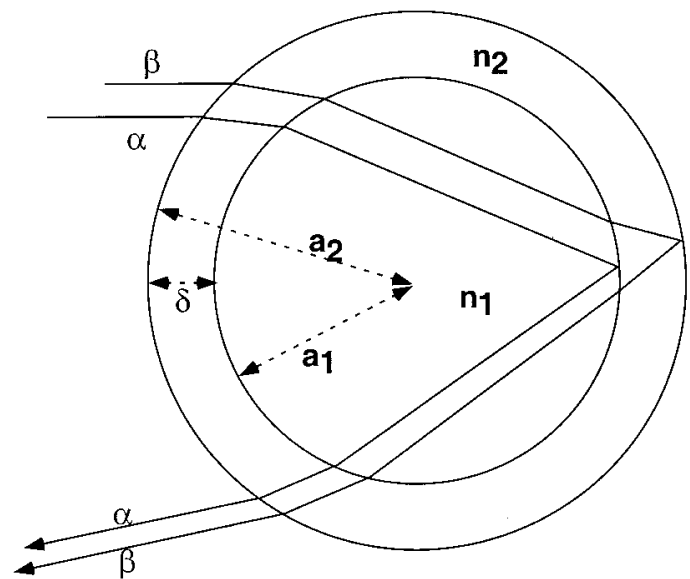

Fig. 1. Coated particle geometry. The inner radius is $a_{1}$ and the coating thickness is $\delta$. The core index of refraction is $n_{1}$ and the coating index is $n_{2}$. The ray paths creating the $\alpha$ and $\beta$ rainbows are indicated on the diagram.

before to our knowledge. We discuss the relevance of our observations to the practical aspects of rainbow refractometry in our conclusions.

To observe these phenomena, one needs to be able to examine a system in which the coating thickness can be easily varied by more than an order of magnitude. Although a test tube filled with water makes a good coated cylinder, it is not ideally suited to this study because the relative thickness of the coating (the glass) is a significant fraction of the core diameter (the water): typically of the order of 5-20\%. With this thickness, one can easily see the twin rainbows separated by $\sim 5-10^{\circ}$. However, if one wishes the coating to act as a thin film, the relative thickness of the coating to the core must be at least an order of magnitude smaller. In the literature there has already been some study of rainbow scattering by liquids in beakers: Hattori et al. did this in their development of a rainbow refractometry system to measure the refractive index of a liquid. ${ }^{19-21}$ In this study the researchers had to account for the thickness of the glass beaker holding their liquid sample to measure the index of the liquid accurately. To do this, they found the position of what we call the $\beta$ rainbow by using a simple raytracing program. However, these researchers did not observe the twinning of the primary rainbow into two separate bows because they did not consider the possibility of twinned rainbows in their study. (It must be pointed out here that the $\alpha$ rainbow is dim under these conditions and not easily seen unless one is looking for it.) Along similar lines, Marcuse and Presby used rainbow scattering by a step-index optical fiber to measure core and cladding diameters and to investigate core-cladding concentricity. ${ }^{34,35}$ Their research forms a complement to this paper: The experimental conditions they considered (small core, large coating) are the reverse of those considered here. Interestingly enough, they also saw the doubling of the primary rainbow because of refraction in the core, although for different reasons than the twinning observed here. They did not systematically vary the coating diameter in their research, as this is extremely difficult to do by use of optical fiber.

In this paper we investigate rainbow scattering by a thin liquid coating on a transparent cylinder. This coating can be made when the end of a transparent cylindrical rod is dipped into a liquid such as water. As the liquid coating sags under the influence of gravity, its thickness varies continuously over time. This allows us to examine the coating near the bottom end of the rod as it progressively thins from over $100 \mu \mathrm{m}$ to approximately $1 \mu \mathrm{m}$. By doing this, we can observe several phenomena predicted in the paper by Lock et $a l .{ }^{33}$ : the twinning of the primary bow when the coating is thick and thin-film effects when it is thin enough that the twin rainbows merge. Because both depend sensitively on the thickness of the liquid layer, this technique offers a method for measurement of thinfilm thicknesses to high accuracy. We discuss the promise of this technique and some experimental problems associated with it in the conclusions.

\section{Rainbow Scattering by a Coated Sphere}

Before we describe our experimental system and results, it is useful to review the theory of rainbow scattering by a coated sphere. ${ }^{33}$ Figure 1 shows the geometry that we consider. As stated above, $n_{1}$ and $a_{1}$ are the index and radius of the core, and $n_{2}$ and $a_{2}$ are those for the coating. $\delta=a_{2}-a_{1}$ is the coating thickness. In ray theory, the rainbow angle is a local minimum in the scattering angle with respect to the impact parameter of a family of parallel rays hitting the sphere. ${ }^{36,37}$ The primary rainbow angle is the minimum for those rays undergoing one internal reflection before exiting, whereas the secondary is created by two, the tertiary by three, etc. (In theory, there is no end to this: rainbows up to the order of 206 have been reported in the optics literature. ${ }^{38,39}$ ) In this paper we consider only the primary rainbow. Because of the two possible ray paths shown in Fig. 1, there are two separate minima in the scattering angle, which we label $\theta_{\alpha}{ }^{R}$ and $\theta_{\beta}{ }^{R}$, that is to say, the coated sphere produces twin primary rainbows.

The angles of the rainbows are functions of the relative thickness of the coating, $\delta / a_{1}$ and the refractive indices of the core and coating. In Ref. 33 the authors derived an approximate formula for $\theta_{\alpha}$ and $\theta_{\beta}$, which is correct to first order in $\delta / a_{1}$. Call $\theta_{0}{ }^{R}$ the scattering angle for the primary rainbow of a homogeneous sphere of index $n_{1}$. Then the scattering angles for the twin rainbows are approximately ${ }^{33}$

$$
\theta_{\alpha}{ }^{R}=\theta_{0}^{R}+\frac{2 \delta}{a_{1}}\left[\left(\frac{4-n_{1}{ }^{2}}{3 n_{2}^{2}+n_{1}{ }^{2}-4}\right)^{1 / 2}-\left(\frac{4-n_{1}{ }^{2}}{n_{1}^{2}-1}\right)^{1 / 2}\right],
$$




$$
\theta_{\beta}{ }^{R}=\theta_{0}{ }^{R}+\frac{2 \delta}{a_{1}}\left[2\left(\frac{4-n_{1}{ }^{2}}{3 n_{2}{ }^{2}+n_{1}{ }^{2}-4}\right)^{1 / 2}-\left(\frac{4-n_{1}{ }^{2}}{n_{1}{ }^{2}-1}\right)^{1 / 2}\right] .
$$

This derivation is based on a Taylor-series expansion of the rainbow angles derived from ray theory. The analysis is not accurate for arbitrarily large or small coating thicknesses. It was checked by use of the Aden-Kerker extensions of Mie theory for a coated sphere. ${ }^{40}$ By doing this, Lock et al. ${ }^{33}$ found that the results given above were valid typically for $10^{-3}<$ $\delta / a_{1}<0.1$. They also found that, for some typical values of $n_{1}, n_{2}$, and $a_{1}$, there was a transition near $\delta / a_{1}=0.3$ where the primary rainbow vanished. For $\delta / a_{1}<10^{-3}$, the situation is more interesting: For these thicknesses, the coating acts as a thin film. Because the twin rainbows emerge at effectively the same scattering angle, they can interfere constructively or destructively, depending on the coating thickness. Thus there is a periodic variation in the brightness of the rainbow as the coating thickness varies.

It is useful to use Airy theory to estimate where the coating thickness is thin enough that it acts as a thin film. This will be important for the later evaluation of our experimental results. Let us call the separation of the $\alpha$ and $\beta$ rainbows $\Delta \theta_{\alpha \beta}{ }^{R}$. Then

$$
\Delta \theta_{\alpha \beta}{ }^{R}=\frac{2 \delta}{a_{1}}\left(\frac{4-n_{1}{ }^{2}}{3 n_{2}{ }^{2}+n_{1}{ }^{2}-4}\right)^{1 / 2}
$$

from Eqs. (1a) and (1b). Because the coating is a thin film, the rainbow pattern in the far field will be the superposition of two Airy functions whose parameters depend on only $n_{1}$ and $a_{1}$ (or, equivalently, $x_{1}=$ $2 \pi a_{1} / \lambda$.) The electric field amplitude for either of the rainbows will then be proportional to

$$
E \sim A i\left[-\left(x_{1}^{2 / 3} / h^{1 / 3}\right)\left(\theta-\theta_{0}^{R}\right)\right],
$$

where $E$ is the amplitude of the electric field, $\theta$ is the scattering angle in the far field, $A i(z)$ is the Airy integral, ${ }^{41,42}$ and

$$
h=\frac{\left(p^{2}-1\right)^{2}}{p^{2}} \frac{\left(p^{2}-n_{1}^{2}\right)^{1 / 2}}{\left(n_{1}^{2}-1\right)^{3 / 2}} .
$$

For the primary rainbow, $p=2$ and for $n_{1}=1.5, h=$ 2.13. When the definition given by Abramowitz and Stegun is used, ${ }^{41}$ the first peak of $A i(-z)$ occurs for $z=1.01879$ and the first zero at $z=2.33811$. We use a Rayleigh-like criterion and state that the rainbows cannot be resolved when the angular separation of the two bows is less than the separation of the first maximum and the first minimum of the diffraction pattern. Using Eqs. (1) and (2) and approximation (3), we arrive at

$$
\delta / a_{1}>0.660\left(h^{1 / 3} / x_{1}^{2 / 3}\right)\left(\frac{3 n_{2}^{2}+n_{1}^{2}-4}{4-n_{1}^{2}}\right)^{1 / 2}
$$
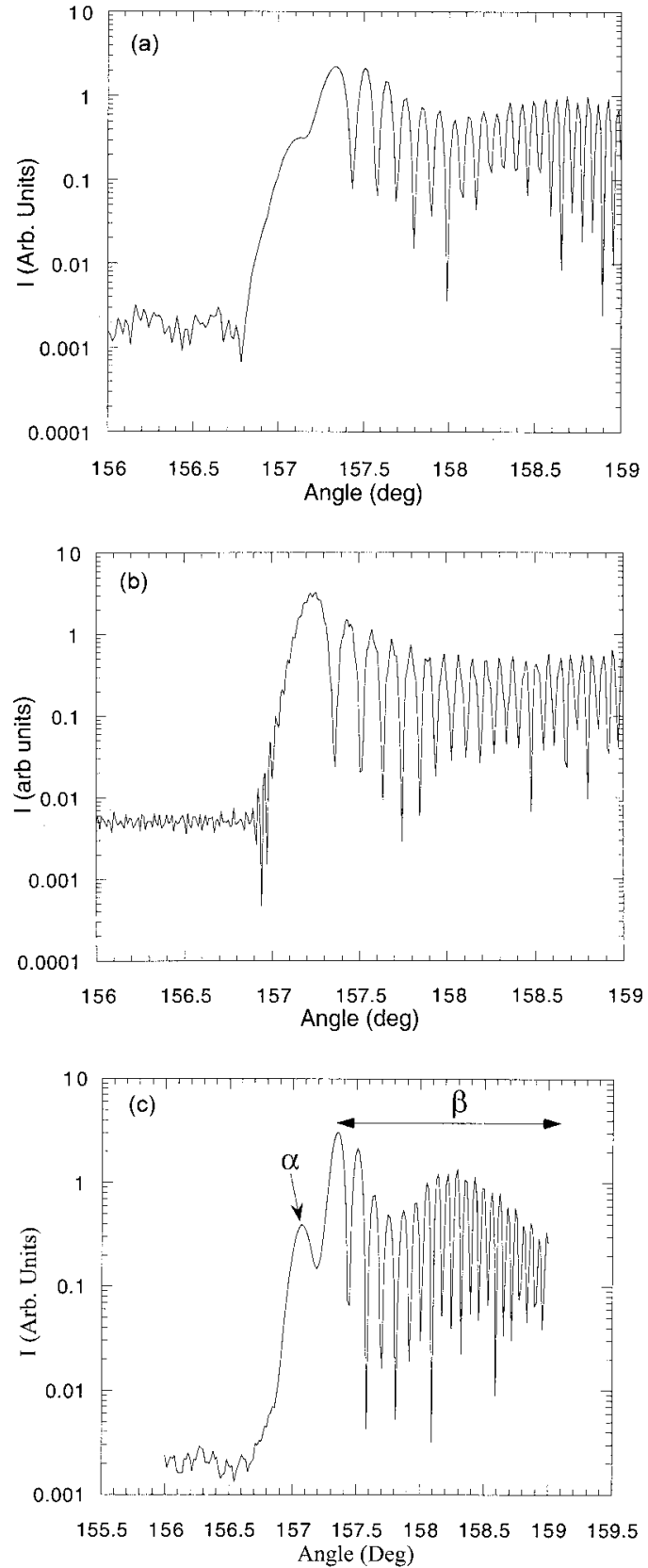

Fig. 2. Theoretical values for the scattering intensity near the primary rainbow for a coated sphere. The calculations are based on the Aden-Kerker extension to Mie theory. Refractive indices: $n_{1}=1.5, n_{2}=1.33, a_{1}=2.6 \mathrm{~mm}$. The intensity is shown on a linear scale to make a comparison with Fig. 5(b) easier: (a) $\delta / a_{1}=$ $2 \times 10^{-3}$, (b) $\delta / a_{1}=10^{-3}$, (c) $\delta / a_{1}=3 \times 10^{-3}$.

for two separate rainbows to be seen. For $a_{1}=2.6$ $\mathrm{mm}, \lambda=0.6328 \mu \mathrm{m}$ (i.e., $\left.x_{1}=2.6 \times 10^{4}\right), n_{1}=1.5$, and $n_{2}=1.33$, this corresponds to $\delta / a_{1}>1.35 \times$ $10^{-3}$. This result is extremely close to that of Ref. 33 in which the criterion for the rainbow separation was given as $\delta / a_{1}>10^{-3}$. We can compare these results to the Aden-Kerker theory by calculating the scattering phase function for a coated sphere at angles near the rainbow angle. ${ }^{33}$ Figure 2 shows the intensity of light near the rainbow angle for the parame- 


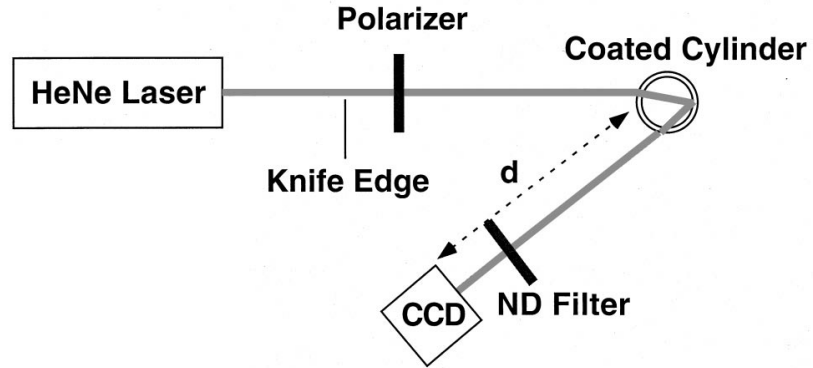

Fig. 3. Experimental diagram. Laser power is $3 \mathrm{~mW}$ and the distance from the cylinder center to the camera is $d=5.6 \mathrm{~cm}$. The laser was polarized parallel to the cylinder axis. The neutraldensity (ND) filter had an optical density of 0.3 .

ters used above. In Fig. 2(a), $\delta / a_{1}=2.0 \times 10^{-3}$, in Fig. $2(\mathrm{~b}), \delta / a_{1}=1.0 \times 10^{-3}$, and in Fig. $2(\mathrm{c}), \delta / a_{1}=$ $3 \times 10^{-3}$. As can be seen, the criterion works well for predicting the coating thickness where the rainbows merge.

\section{Experimental Setup and Results}

\section{A. Singly Coated Cylinder}

Figure 3 shows the experimental setup used in this experiment. The unexpanded beam from a $\mathrm{He}-\mathrm{Ne}$ laser is incident on the coated cylinder at an angle near the impact angle of the rainbow ray. Because the beam is not expanded, considerable power can be channeled into the rainbow, making the phenomena we report here considerably easier to observe than if the beam uniformly illuminated the cylinder. In addition, this eliminates interferences between the rainbow and the reflections off of the surface of the cylinder. We also have a knife edge that can be inserted into the beam to eliminate extraneous rays that do not contribute to the formation of rainbows. This beam blocking proved useful in the investigation of the doubly coated cylinder (see Subsection 3.B).

The beam was polarized parallel to the cylinder axis. The CCD camera was set up so that the primary rainbow was normally incident on its active surface at a distance of $d=5.6 \mathrm{~cm}$ from the center of the rod. We did not use a lens on the camera. The video signal was captured on a computer for analysis.

In this experiment, the end of a glass rod (typically, a stirring rod used for mixing chemicals) was dipped in water and pulled out. We made a movie of the twin rainbows as the coating thickness changed as the water pooled up at the end of the rod under the influence of gravity. The rainbows were measured approximately $5 \mathrm{~mm}$ above the bottom end of the rod, where a water film could persist for longer than 6 min. The diameter of the stirring rod is $d=0.485$ $\mathrm{mm}$, and it had an index of refraction of $n_{1}=1.502 \pm$ 0.03 , which was determined by rainbow refractometry of the uncoated cylinder. ${ }^{43}$ For the rest of the paper we assume $n_{1}=1.5$ and $n_{2}=1.33$ when we evaluate our data.

When making a movie, we would dip the rod into distilled water to a depth of a few centimeters and

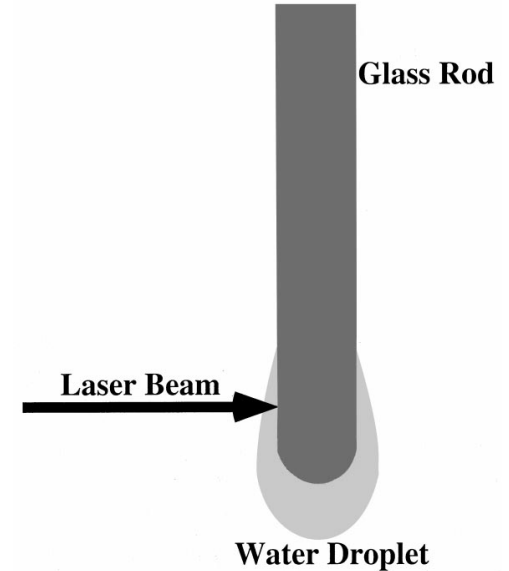

Fig. 4. Diagram of a typical pendant droplet investigated in the experiment. The cylinder diameter is $2 a_{1}=5.2 \mathrm{~mm}$. The laser beam is incident a distance roughly $5 \mathrm{~mm}$ above the end of the rod.

then lift the rod out. The behavior of the water coating on the rod is complicated. Typically, a pendant droplet would form on the end of the rod and drop off within a few seconds. Following that, a second pendant droplet would form and hang on to the end of the rod for times of up to several minutes. In our experiments, we waited until after the first droplet fell off to start taking data. As the pendant drop forms, the water coating on the rod itself decreases continually as water pooled onto the droplet under the influence of gravity. Figure 4 is a diagram of the rod with a typical pendant droplet hanging off of it. Because of the continual thinning of the water layer, the twin rainbows would approach each other and eventually merge. Following this, the rainbow will then dim and brighten periodically because of the interference of the two bows as the water layer continued to thin.

The movie we made for our experiment lasted approximately $5.5 \mathrm{~min}$ before the pendant droplet fell off the end of the rod. The twin rainbows are visible separately for the first $4 \mathrm{~min}, 28 \mathrm{~s}$ of filming: During the remaining minute, the effect of the continually thinning water film can be seen as the rainbow brightens and dims because of constructive and destructive interference of the rainbows.

Figure 5(a) is a digitized image of the rainbow approximately $1 \mathrm{~min}$ into the experiment. As can be seen, two rainbows are clearly visible. Because the water-glass interface is optically softer than the interface between water and air, the $\beta$ rainbow is brighter than the $\alpha$ rainbow. Figure 5(b) shows the intensity profile of the two rainbows. The angular separation of the two bows in this frame is $0.8^{\circ}$. The intensity profile was a vertical average of the data in the highlighted box in Fig. 5(a). Note that there is a moiré interference pattern visible in the supernumerary region in this picture. The interference pattern is due to gravitational sagging of the water film: The coating thickness at the bottom is thicker than at the top, so the separation of the two rainbows (and 

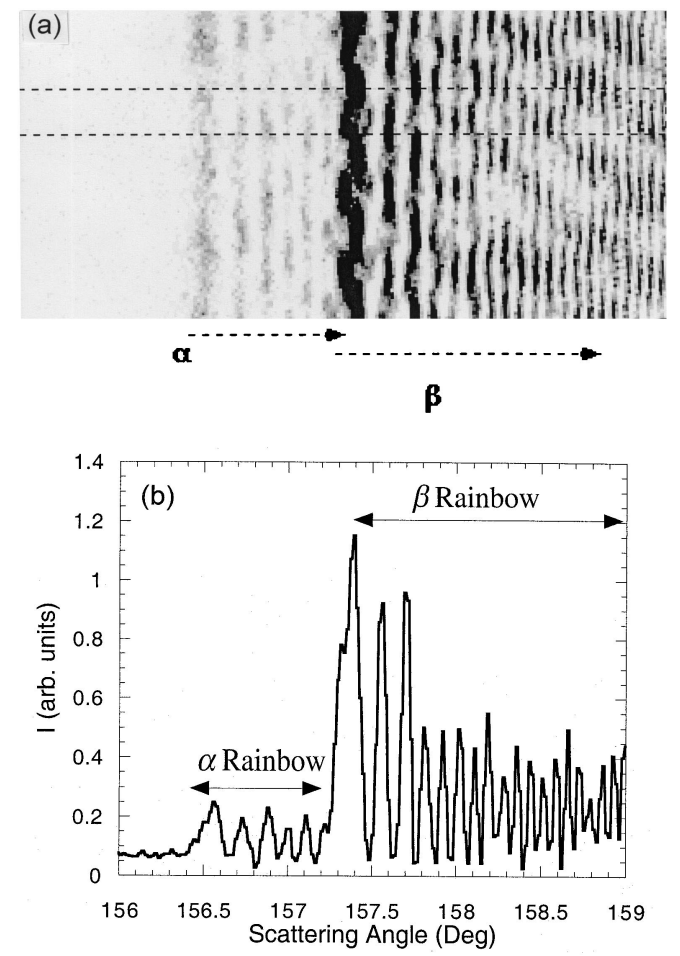

Fig. 5. (a) Digitized image of the twin rainbows. $\delta / a_{1} \sim 10^{-2}$. (b) Intensity profile of data from image (a). Angular separation of the rainbows is $0.8^{\circ}$.

hence the phase difference between them) is a function of height.

Figure 6 is a graph of the angular deviation of each bow from $\theta_{0}^{R}$ as a function of time, for as long as the twin bows were visible. After the first few seconds, the angular deviation decreases exponentially over time, indicating that the coating thickness decreases exponentially. Although it is not immediately apparent from Fig. 6, the angular separation of the twin bows decreases exponentially as well. From Eqs.

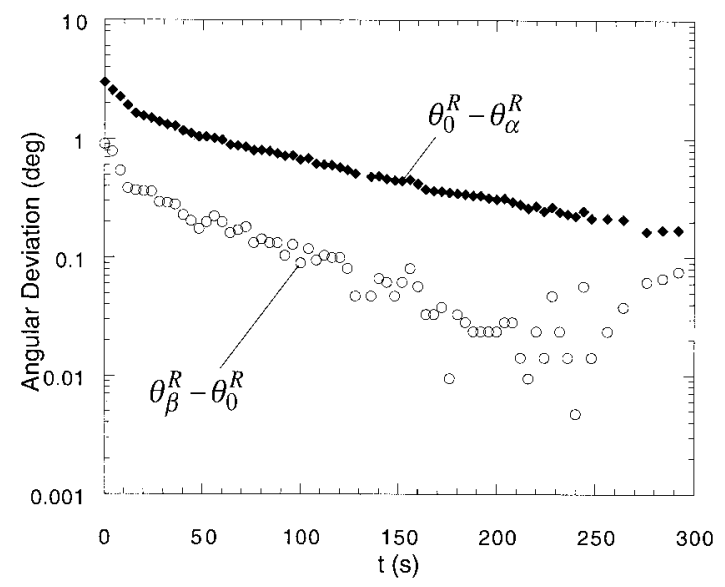

Fig. 6. Angular deviation of each bow from $\theta_{0}{ }^{R}$ as a function of time.

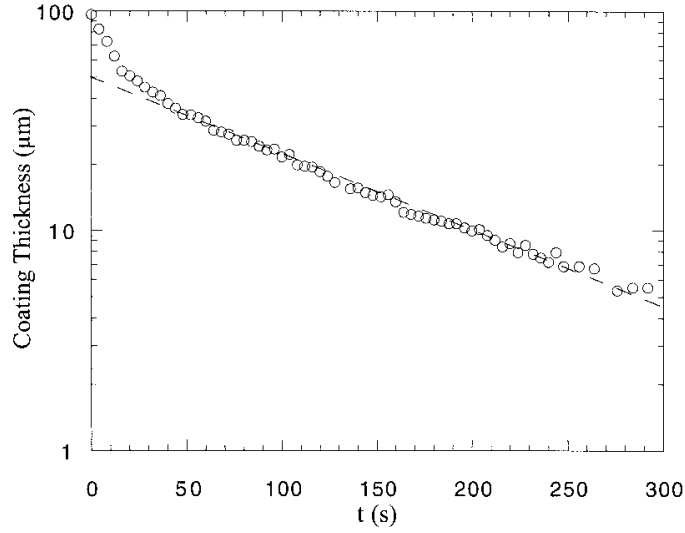

Fig. 7. Calculated value of the coating thickness as a function of time by use of the data from Fig. 6 .

1(a) and 1(b), we can derive an equation for the coating thickness:

$$
\delta^{\text {th }}=\frac{a_{1}}{2}\left(\frac{3 n_{2}{ }^{2}+n_{1}{ }^{2}-4}{4-n_{1}{ }^{2}}\right)^{1 / 2}\left(\theta_{\alpha}{ }^{R}-\theta_{\beta}{ }^{R}\right) .
$$

We refer to this as a theoretical value for the coating thickness because we do not have an independent measurement of it, although we believe that it is at worst in error by approximately $25 \%$. We discuss this point below. Figure 7 is a graph of $\delta^{\text {th }}$ as a function of time. The coating thickness decreases from approximately $80 \mu \mathrm{m}$ to approximately $4 \mu \mathrm{m}$; for thicknesses below $4 \mu \mathrm{m}$, the coating was thin enough that the twin rainbows could not be distinguished. After the first several seconds, the coating thickness decreased exponentially in time, with a time constant of $118 \mathrm{~s}$.

Although we do not have an independent measurement of $\delta$, we can apply the following check using the rest of the data from our movie (i.e., from times following the merging of the twin rainbows.) Alternate dimming and brightening of the rainbow correspond to coating thicknesses giving destructive and constructive interference between the two bows. Figure 8 shows the intensity of the first Airy peak of the primary bow as a function of time, showing just this effect. If we assume that the coating continues to thin exponentially with the same time constant as determined above, we can predict the values of the coating thicknesses at the intensity maxima and minima. We can then determine the change in coating thickness between a bright fringe and a dark fringe. From our data, this value is $0.15 \pm 0.04 \mu \mathrm{m}$.

The theoretical value for this change can be calculated by elementary considerations: Using Eq. (19)(23) of Ref. 33, we find that, to change a bright fringe into a dark fringe, the coating layer thickness should change by $0.145 \mu \mathrm{m}$, close to the value we predict based on our data. This leads us to believe that we are measuring the coating thickness accurately. We return to this point in the conclusions.

Finally, Fig. 9(a) is a digitized image of the rainbow after the two bows have merged (approximately 5 


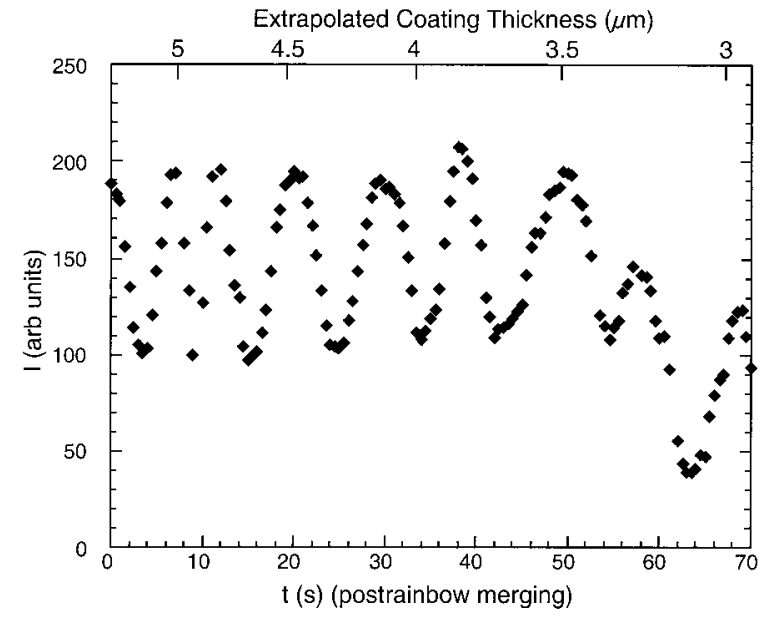

Fig. 8. Intensity of the main rainbow peak as a function of time (after the rainbows merged). The top scale is an interpolated value for the coating thickness by use of the data from Fig. 7.

min into the experiment). Figure 9(b) shows the intensity profile of the rainbow. The intensity profile was a vertical average of the intensity in the area enclosed by the dashed lines in Fig. 9(a). If we view Fig. 9 as being due to the interference between two rainbows whose angular separation is too small to resolve, it is clear from the spatial pattern that the phase difference between the bows is a function of both height and scattering angle.
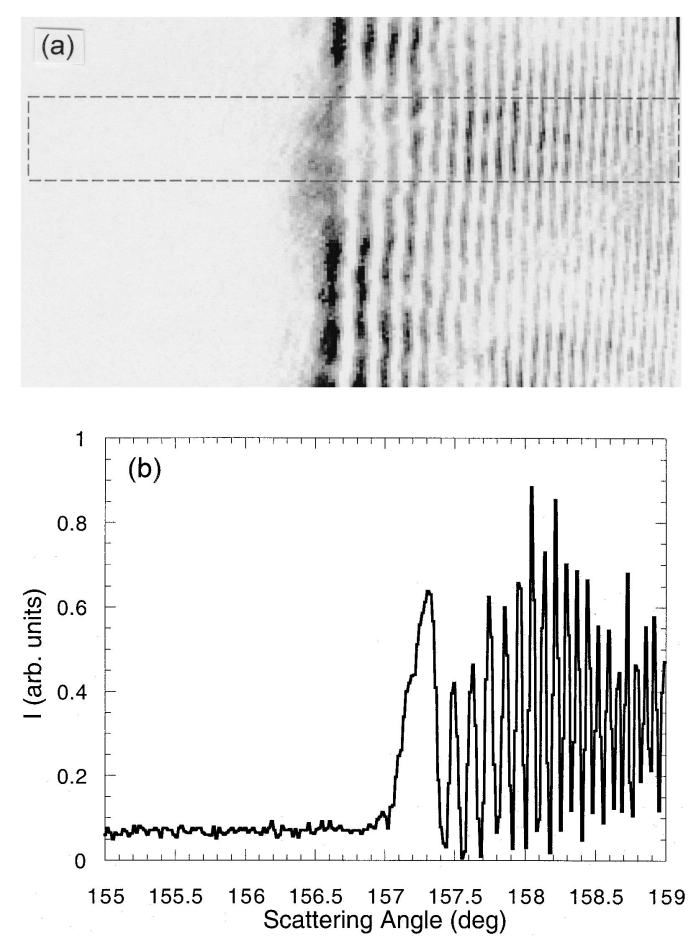

Fig. 9. (a) Digitized image of the rainbow at $t=30 \mathrm{~s}$ after the merging. (b) Intensity profile of data from image (a). The intensity profile was made from the area enclosed by the dashed lines in (a).

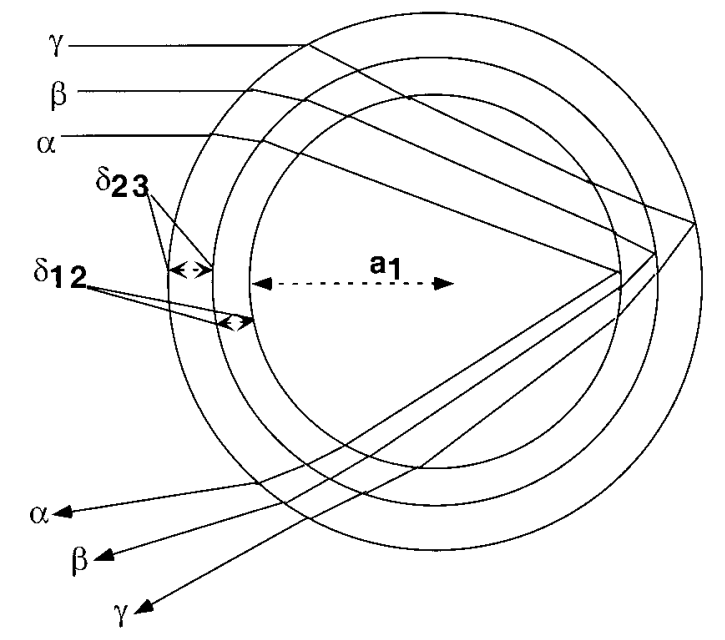

Fig. 10. Doubly coated particle geometry. Core index, $n_{1}$; coating 1 index, $n_{2}$; coating 2 index, $n_{3}$. The hypothesized ray paths for the $\alpha, \beta$, and $\gamma$ rainbows are shown.

\section{B. Doubly Coated Cylinder}

Multiply coated particles present a much greater theoretical challenge than ones with a single coating. However, they are relatively easy to make, but not so easy to understand. In our laboratory we took a pipet tube filled with water and dipped its end into olive oil to obtain a doubly coated cylinder. Figure 10 shows the geometry that this represents. The index of refraction of the glass is approximately 1.5, whereas that of olive oil is $1.47 \pm 0.02$, measured by rainbow refractometry on a pendant droplet. For this experiment, $a_{1}=0.25 \mathrm{~cm}, \delta_{1}=0.1 \mathrm{~cm}$, and $\delta_{2}$ varied over time as the olive oil dripped off the rod.

For this experiment, the angular positions of the $\alpha$ and $\beta$ rainbows were determined before we coated the end of the test tube. These are $\theta_{\alpha}{ }^{R}=108.8^{\circ}$ and $\theta_{\beta}{ }^{R}=125.2^{\circ}$. Because the coating thickness is a significant fraction of the core thickness, we cannot use Eqs. 1(a) and 1(b) to predict the twin-rainbow positions. Using our coated sphere scattering program, we find $\theta_{\alpha}^{R}=107^{\circ}$ and $\theta_{\beta}{ }^{R}=128^{\circ}$, which is close to the measured values.33 Any deviation is probably due to uncertainty in the index of refraction and the exact shape of the test tube.

We expect to see three primary rainbows (triplets) from the ray paths highlighted in Fig. 10. These are due to reflections from the air-glass, glass-oil, and oil-air interfaces (labeled $\alpha, \beta$, and $\gamma$ ). We therefore expect that the $\gamma$ rainbow will be brightest, followed by the $\alpha$ rainbow, whereas the $\beta$ rainbow will be dim because of the near match of the oil and glass indices. We also expect, on physical grounds, that the $\alpha$ rainbow will be near the $\alpha$ rainbow angle for the uncoated water-filled test tube, whereas the $\beta$ and $\gamma$ rainbows will be near the $\beta$ rainbow. We did indeed observe three rainbows in the right position, one much dimmer than the other two. Figure 11 is a video image of the three rainbows. The dimmest rainbow was the $\beta$ rainbow, located between the $\alpha$ and $\gamma$ bows. Figure 12 shows the positions of the three rainbows 


\section{Scattering Angle}

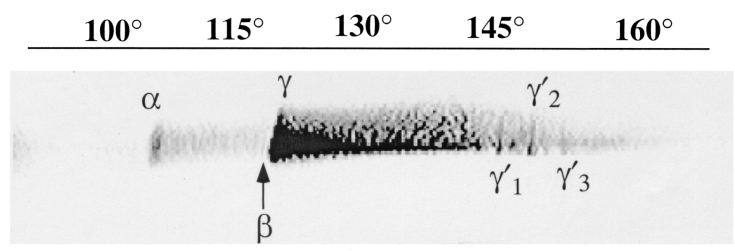

Fig. 11. Video image of the triplet rainbows $(\alpha, \beta$, and $\gamma)$ and the three $\gamma^{\prime}$ rainbows. The $\beta$ rainbow is almost invisible on camera, but its position is indicated.

as a function of time. For these experiments, the angular separation of the three rainbows was too large to capture directly on the camera face. Instead, they were projected onto a viewing screen, which was imaged onto the camera. In Fig. 12, and in Fig. 13, the angular displacements of the triplet rainbows are measured from the center of the visual field of the camera. As the olive oil layer thinned, the $\beta$ and $\gamma$ rainbows merged, whereas the $\alpha$ rainbow stayed separate from the two. This is the expected behavior: As the second coating thins, the rainbow angles should approach the scattering angles for a singly coated cylinder.

We also observed three additional primary rainbows at a larger scattering angle (see Fig. 11). We refer to these as the $\gamma^{\prime}$ family of rainbows, for reasons explained below. They are labeled as $\gamma_{1}{ }^{\prime}, \gamma_{2}{ }^{\prime}$, and $\gamma_{3}{ }^{\prime}$, from the smallest to the largest scattering angle. These are almost certainly due to multiple reflections inside the coating, as discussed below. They are not easily seen (being very dim) unless a beam-blocking technique is used to eliminate background light. ${ }^{43,44}$ The same beam-blocking technique can be used to establish that they are some form of the primary rainbow: As the beam is progressively blocked by a knife edge, the $\alpha, \beta$, and $\gamma$ rainbows vanish once the incoming rays are blocked at their impact parameters. The $\gamma^{\prime}$ rainbows vanish at almost exactly the same values of the impact parameter.

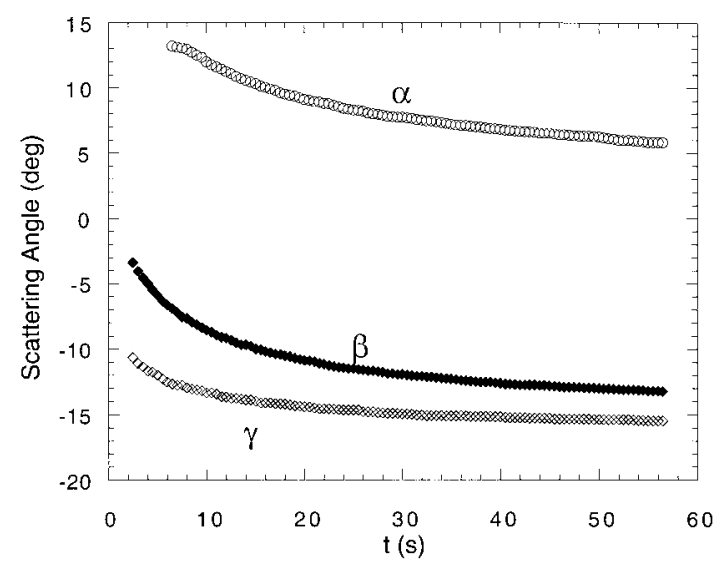

Fig. 12. Angular position of the $\alpha, \beta$, and $\gamma$ rainbows as a function of time. The angle is measured from the center of the visual field on the camera.

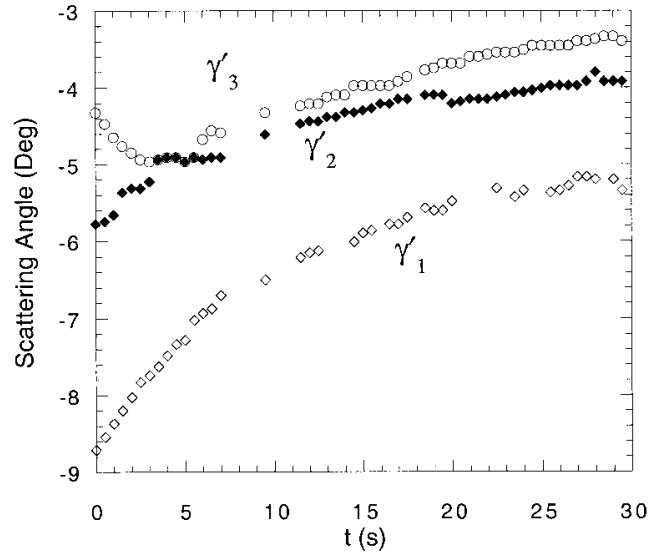

Fig. 13. Angular position of the $\gamma^{\prime}$ complex of rainbows as a function of time. The angle is measured from the center of the visual field on the camera. After $t=3 \mathrm{~s}$, the $\gamma_{2}{ }^{\prime}$ and $\gamma_{3}{ }^{\prime}$ rainbows appear to pass through each other, making the identification of either one ambiguous.

Figure 13 shows the position of these rainbows as a function of time. The same general behavior can be seen as for the $\alpha, \beta$, and $\gamma$ rainbows, except for two things: First, the displacement of the rainbows as the coating thins is generally in the opposite direction of the $\alpha, \beta$, and $\gamma$ bows. This is consistent with these bows being due to additional reflections of rays inside the coating. Second, the $\gamma_{2}{ }^{\prime}$ and $\gamma_{3}{ }^{\prime}$ rainbows seem to move past each other after $t=3 \mathrm{~s}$, although it is extremely difficult to tell which rainbow is which at this point. As the coating layer thinned, these three rainbows asymptotically approached a scattering angle of $144.5^{\circ}$.

\section{Discussion of Results}

\section{A. Singly Coated Cylinder}

It is obvious that the results presented in this paper are in good qualitative agreement with previous theoretical predictions. We observed two of the three regimes predicted in Ref. 33: coating thicknesses large enough to see twin-rainbow formation and thinfilm behavior for $\delta / a_{1} \sim 10^{-3}$ or less. Although we do not have a direct check on the quantitative agreement with theory, our indirect check indicates that we are probably measuring the coating thickness reasonably accurately. However, here we discuss the following possible sources for error and inaccuracy in these measurements:

(1) Discretization error. There is a \pm 1.5 pixel uncertainty in measurement of the position of the main Airy peak for either rainbow. This corresponds to an uncertainty in the angle of the rainbow of $\pm 0.01^{\circ}$. The width of the main Airy peak itself is approximately $0.1^{\circ}$, much broader than the measurement uncertainty. One other point: In this paper we use the position of the maximum of the main Airy peak as the rainbow angle; this is not quite correct, as the Airy peak differs slightly from the position of the 
rainbow as determined by geometrical optics. ${ }^{36,37,42}$ Although the theory of rainbow scattering for a coated cylinder has not been worked out in wave theory, the angular correction for this should be roughly the same for both rainbows. Because the thickness was computed by use of the angular difference between the twin bows, the deviations will cancel out.

(2) The rainbow angle as predicted by geometrical optics is exactly correct only infinitely far away from the cylinder. Because we took all the measurements at a distance of approximately 20 cylinder radii, there should be a small correction to each rainbow angle because of the curvature of the twin-rainbow caustics. We did not make this correction because the shapes of the rainbow caustics of a coated cylinder have not been worked out.

(3) The cross section of the cylinder is not exactly circular. We measured an ellipticity parameter for this cylinder of $5.4 \times 10^{-3}$ using rainbow refractometry. ${ }^{43}$ The ellipticity parameter quoted is in fact a value measured near the center of the rod (in the vertical direction), whereas the coated rainbows are all measured near the end of the rod. Because the end of the rod was cut and melted to round it off, the ellipticity of the rod near the end may be higher than in the center. In addition, the water layer may not be precisely concentric with the glass cylinder. Surface inhomogeneities and the oscillation of the water droplet may change the water layer thickness by a good deal. As above, the theory for the position of the twin bows exists for only two concentric cylinders with a circular cross section.

For these reasons, we believe that our measurements are currently good only to approximately $25 \%$. We will be starting several experiments on optical quality glass cylinders with nearly perfect circular cross sections in the near future. We are also in the process of designing an experiment to allow us to measure the thickness of the coating layer independently.

\section{Doubly Coated Cylinder}

One can find the approximate values for the positions of the $\alpha, \beta$, and $\gamma$ rainbows by the following means: For a given ray path through the particle, we calculated all the angles of reflection and refraction. The deflection angle of the ray with respect to the initial direction of incidence was determined. This expression was differentiated and set to zero to obtain the rainbow condition. Because the expression is a transcendental equation, it cannot be solved analytically. The angle of incidence and all angles of refraction and reflection were determined to first order in the coating thickness and substituted back into the original expression, which was then truncated to first order in coating thickness as well, to obtain the approximate value for the rainbow angles. This is the same method used to find the positions of the $\alpha$ and $\beta$ rainbows in Ref. 33. We define the following quantities: $a$ is the core radius, $\delta_{2}$ is the inner-coating
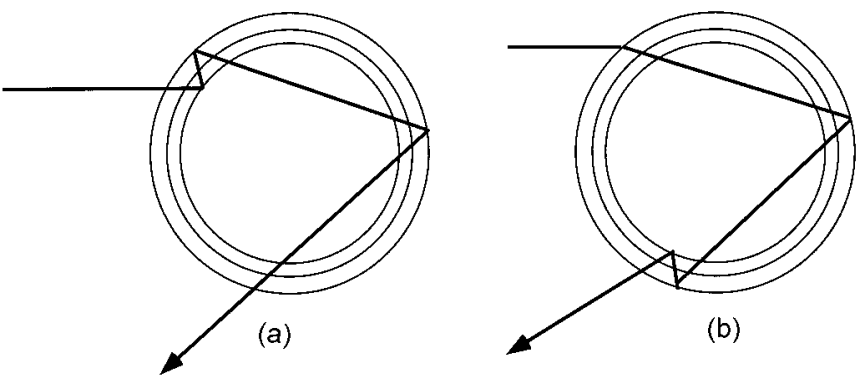

Fig. 14. Ray paths and scattering angles for rainbows that are due to multiple internal reflections inside the coatings. We label these the $\gamma^{\prime}$ paths.

thickness, $\delta_{3}$ is the outer-coating thickness, $n_{1}$ is the core index, $n_{2}$ is the inner-coating index, $n_{3}$ is the outer-coating index, and

$$
\begin{aligned}
M_{21} & =\frac{2\left(4-n_{1}^{2}\right)^{1 / 2}}{\left(3 n_{2}{ }^{2}+n_{1}{ }^{2}-4\right)^{1 / 2}}, \\
M_{31} & =\frac{2\left(4-n_{1}{ }^{2}\right)^{1 / 2}}{\left(3 n_{3}{ }^{2}+n_{1}{ }^{2}-4\right)^{1 / 2}}, \\
N_{1} & =\frac{2\left(4-n_{1}{ }^{2}\right)^{1 / 2}}{\left(n_{1}{ }^{2}-1\right)^{1 / 2}} .
\end{aligned}
$$

The positions of the $\alpha, \beta$, and $\gamma$ rainbows are then

$$
\begin{aligned}
& \theta_{\alpha}{ }^{R}=\theta_{0}{ }^{R}+\frac{\delta_{2}}{a}\left(M_{21}-N_{1}\right)+\frac{\delta_{3}}{a}\left(M_{31}-N_{1}\right), \\
& \theta_{\beta}{ }^{R}=\theta_{0}{ }^{R}+\frac{\delta_{2}}{a}\left(2 M_{21}-N_{1}\right)+\frac{\delta_{3}}{a}\left(M_{31}-N_{1}\right), \\
& \theta_{\gamma}{ }^{R}=\theta_{0}{ }^{R}+\frac{\delta_{2}}{a}\left(2 M_{21}-N_{1}\right)+\frac{\delta_{3}}{a}\left(2 M_{31}-N_{1}\right) .
\end{aligned}
$$

We do not expect Eq. (8) to be accurate for the experiment we are doing here, as $\delta_{2} / a \sim 10^{-1}$, which is probably too thick for the approximations made to derive it. However, with the combination of indices we gave above $\left(n_{1}=1.33, n_{2}=1.5\right.$, and $\left.n_{3}=1.47\right)$, Eq. (7) and (8) do predict that, as the water layer thins, the three rainbows will move in the same direction. This is what we see in Fig. 12. To test this, we dipped the test tube in water. With $n_{3}=1.33$, the $\beta$ and $\gamma$ rainbows should move in the opposite direction of the $\alpha$ rainbow as the coating thins. This is exactly what can be seen.

What of the other three rainbows that are seen? Figure 14 shows two possible ray paths involving a second internal reflection between the core-innercoating and outer-coating-air interfaces. We label these the $\gamma^{\prime}$ rainbows. The scattering angle for the rainbows produced by each of the different ray paths is

$$
\theta_{\gamma^{\prime}}{ }^{R}=\theta_{0}{ }^{R}+\frac{\delta_{2}}{a}\left(3 M_{21}-N_{1}\right)+\frac{\delta_{3}}{a}\left(3 M_{31}-N_{1}\right) .
$$


(Because of the circular symmetry that we assumed in deriving these expressions, the two different ray paths lead to rainbows emerging at the same angle.) We believe that the reflections leading to the additional rainbows seen in this experiment are due to these paths. In the limit $\delta_{3} \ll \delta_{2}$, the scattering angle for the $\gamma^{\prime}$ rainbows approaches $141.5^{\circ}$ in theory, which is in reasonable agreement with the asymptotic value of $144.5^{\circ}$ that we measured. Although there are other ray paths involving internal reflections leading to rainbows near these scattering angles, we do not believe that they produce the three rainbows we see, as all of them produce a rainbow that is an order of magnitude dimmer than the $\gamma^{\prime}$ ray paths. Why we see three rainbows and why two appear to merge while one stays at an approximately constant angular distance from the other two rainbows is unknown.

\section{Conclusions}

We have reported the first detailed study to our knowledge of rainbow scattering by coated particles in a system in which the coating thickness can be varied by nearly 2 orders of magnitude. To our knowledge, this is the first experimental study ever made of a doubly coated particle under any condition. There is a good deal of interest in such experimental studies because of rainbow refractometry. Although the coated cylinder cannot mimic all the behavior of a coated droplet, as it is essentially a two-dimensional scatterer, there is much that can be done with it. As mentioned above, there have been several theoretical studies of rainbow scattering by radially inhomogeneous spheres. ${ }^{27-31}$ Comparison of these studies with experiment can be done with a properly fabricated inhomogeneous cylinder with a circular cross section. Research such as this opens up a promising new method for testing the practicality of rainbow refractometry.

In addition, it also presents a promising method for measuring the index of refraction and thickness of thin liquid films and associated parameters such as temperature and the contact angle of a pendant droplet. The experiments we performed here indicate that the separation of the rainbows can be used to measure coating thicknesses as little as a few micrometers thick. However, this is not all: After the rainbows merge, the thin-film interference effects should allow measurements of films as thin as approximately $0.5 \mu \mathrm{m}$. We have dubbed this technique twin-rainbow metrology and have begun a series of experiments designed to test its practicality for the accurate measurement of thin liquid and solid films. The results of this research will be presented in a future paper.

A further point is that the complicated twodimensional moiré pattern that is due to the interference of the twin bows contains much information about the vertical gradient of the coating thickness. To examine this further, we projected the twin rainbows (from a water-coated rod) onto a screen and imaged them by using the CCD camera. To view a

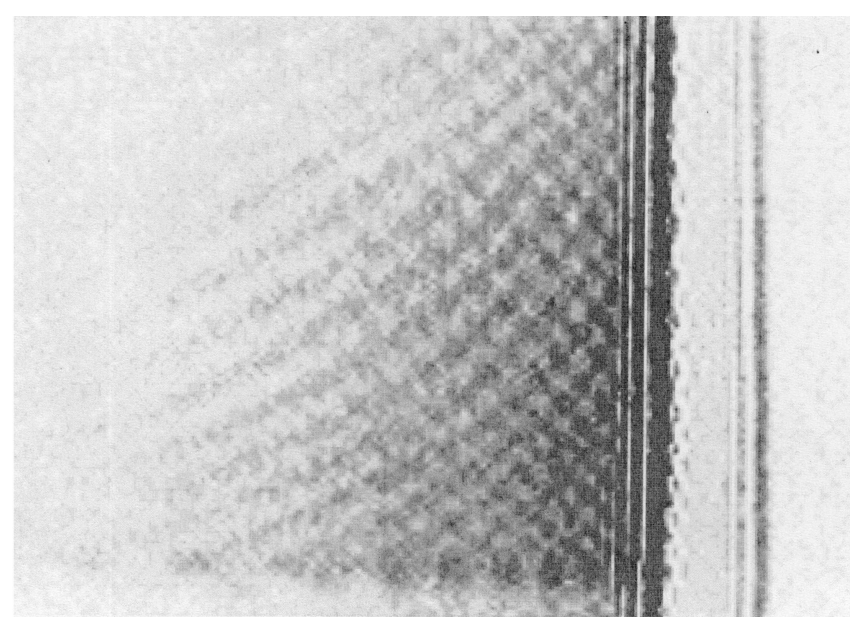

Fig. 15. Video image of a large vertical section of the twin rainbows from a water-coated glass cylinder. The water coating is thicker at the bottom because of gravitational sagging of the water layer.

large vertical section of the rod, we expanded the laser beam to a length of $4 \mathrm{~cm}$ by using a cylindrical lens. Figure 15 shows the twin-rainbow image. The fact that the water layer is thicker near the bottom of the rod is clear from the separation of the rainbows.

We tried to simulate the moiré pattern by calculating the far-field diffraction pattern as the square of the superposition of two Airy integrals with a coatingthickness-dependent phase shift between them. If one normalizes coordinates so that $z=\left(x_{1}{ }^{2 / 3} / h^{1 / 3}\right)$ $\left(\theta_{\alpha}{ }^{R}-\theta_{0}{ }^{R}\right)$ and $\Delta z=\left(x_{1}{ }^{2 / 3} / h^{1 / 3}\right) \Delta \theta_{\alpha \beta}$, a simple theory based on the Airy approximation predicts that

$$
\begin{aligned}
I(z)= & {\left[c_{\alpha} A i(-z)\right]^{2}+\left\{c_{\beta} A i\left[-\left(z-\Delta z_{\alpha \beta}\right]\right\}^{2}\right.} \\
& +2 c_{\alpha} c_{\beta} A i(-z) A i[-(z \\
& \left.\left.-\Delta z_{\alpha \beta}\right)\right] \cos \left[\frac{n_{2}\left(x_{1} h_{1}\right)^{1 / 3}}{\sin \phi_{R}{ }^{2}} \Delta z_{\alpha \beta}\right],
\end{aligned}
$$

where $I(z)$ is the intensity of the far-field diffraction pattern, $c_{\alpha}$ and $c_{\beta}$ are the amplitudes of the $\alpha$ and $\beta$ rainbows, and

$$
\sin \phi_{R}^{2}=\left(\frac{4-n_{1}^{2}}{3 n_{2}^{2}}\right)^{1 / 2}
$$

Figure 16 shows the results of one of these simulations. $\Delta z_{\alpha \beta}$ changes linearly from 10 at the top of Fig. 16 to 11 at the bottom. The values of $x_{1}, n_{1}$, and $n_{2}$ were chosen to match our experimental conditions. The amplitudes of the Airy functions and the relative displacements were chosen to match Fig. 15 as best as we could. The results are suggestive, indicating that we may be able to interpret the moiré interference pattern using a simple theory. In particular, the shape of the bright and dark bands in Figs. 15 and 16 are remarkably similar. We will present a detailed analysis of the interference pattern in a future paper. 


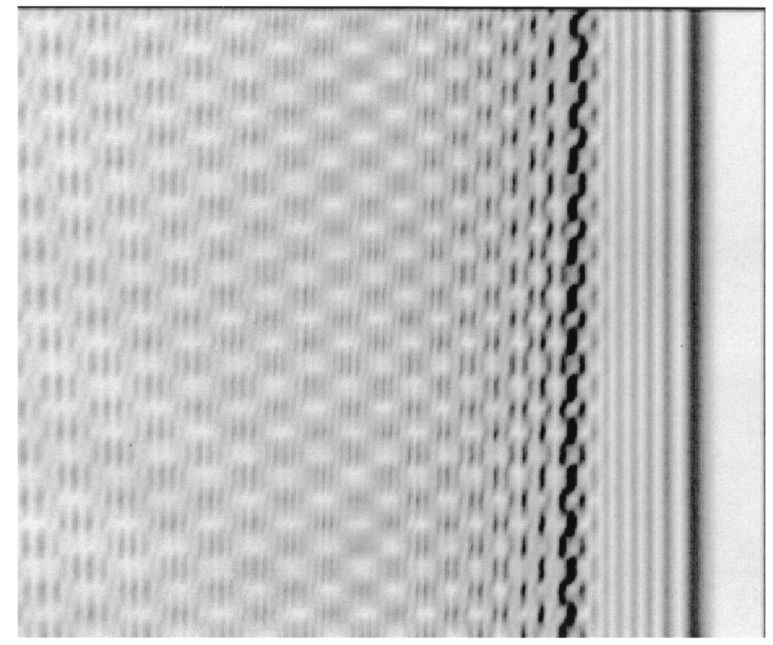

Fig. 16. Computer simulation of the moire interference pattern shown in Fig. 15. Vertical and horizontal scales are in normalized units.

This research was supported by National Science Foundation grant PHY-9987862 and by NASA grant NCC3-521. We thank Chris Mattia for his help on our videos; and Ruth-Anne Armitage, Darlene Van Gaasbeck, Al Hovland, and Andy Koch for providing us with supplies. We also thank our reviewers for several good suggestions about the paper and for calling our attention to the work of Marcuse and Presby. We dedicate this research to Alexandra Adler, who made her appearance while it was in progress.

\section{References}

1. W. Mobius, "Zur Theorie des Regenbogens und ihrer experimentallen Prufung," Abh. Math.- Phys. Kl. Saechs. Ges. Wiss. 30, 105-254 (1907-1909).

2. W. Mobius, "Zur Theorie des Regenbogens und ihrer experimentallen Prufung," Ann. Phys. (Leipzig) 33, 1493-1558 (1910).

3. A. B. Fraser, "Why can the supernumerary bows be seen in a rain shower?," J. Opt. Soc. Am. 73, 1626-1628 (1983).

4. D. Marcuse, "Light scattering from elliptical fibers," Appl. Opt. 13, 1903-1905 (1974).

5. P. L. Marston, "Rainbow phenomena and the detection of nonsphericity in drops," Appl. Opt. 19, 680-685 (1980).

6. P. L. Marston and E. H. Trinh, "Hyperbolic umbilic diffraction catastrophe and rainbow scattering from spheroidal drops," Nature (London) 312, 529-531 (1984).

7. P. L. Marston, "Cusp diffraction catastrophe from spheroids: generalized rainbows and inverse scattering," Opt. Lett. 10, 588-590 (1985).

8. J. F. Nye, "Rainbow scattering from spheroidal drops-an explanation of the hyperbolic umbilic foci," Nature (London) 312, 531-532 (1984).

9. J. F. Nye, "Rainbows from ellipsoidal water drops," Proc. R. Soc. London, Ser. A 438, 397-417 (1992).

10. K. Sassen, "Optical backscattering from near-spherical water, ice, and mixed phase drops," Appl. Opt. 16, 1332-1341 (1977).

11. G. Kaduchak and P. L. Marston, "Hyperbolic umbilic and $E_{6}$ diffraction catastrophes associated with the secondary rainbow of oblate water drops: observations with laser illumination,” Appl. Opt. 33, 4697-4701 (1994).

12. G. Kaduchak, P. L. Marston, and H. J. Simpson, " $E_{6}$ diffraction catastrophe of the primary rainbow of oblate water drops: observations with white-light and laser illumination," Appl. Opt. 33, 4691-4696 (1994).

13. J. P. A. J. van Beeck and M. L. Riethmuller, "Nonintrusive measurements of temperature and size of single falling raindrops," Appl. Opt. 34, 1633-1639 (1995).

14. J. P. A. J. van Beeck and M. L. Riethmuller, "Rainbow phenomena applied to the measurement of droplet size and velocity and to the detection of nonsphericity," Appl. Opt. 35, 22592266 (1996)

15. J. van Beeck, "Rainbow phenomena: development of a laserbased, non-intrusive technique for measuring droplet size, temperature and velocity," Ph.D. dissertation (Technische Universiteit Eindhoven, Eindhoven, The Netherlands, 1997).

16. P. Massoli, F. Beretta, A. D’Alessio, and M. Lazzaro, “Temperature and size of single transparent droplets by light scattering in the forward and rainbow regions," Appl. Opt. 32, 32953301 (1993).

17. C. W. Chan and W. K. Lee, "Measurement of a liquid refractive index by using high-order rainbows," J. Opt. Soc. Am. B 13, 532-535 (1996).

18. X. Han, K. F. Ren, Z. Wu, F. Corbin, G. Gouesbet, and G. Gréhan, "Characterization of initial disturbances in a liquid jet by rainbow sizing," Appl. Opt. 37, 8498-8503 (1998).

19. H. Hattori, H. Yamanaka, H. Kurniawan, S. Yokoi, and K. Kagawa, "Using minimum deviation of a secondary rainbow and its application to water analysis in a high-precision, refractive-index comparator for liquids," Appl. Opt. 36, 55525556 (1997).

20. H. Hattori, H. Kakui, H. Kurniawan, and K. Kagawa, "Liquid refractometry by the rainbow method," Appl. Opt. 37, 41234129 (1998).

21. H. Hattori, "Simulation study on refractometry by the rainbow method," Appl. Opt. 38, 4037-4046 (1999).

22. N. Roth, K. Anders, and A. Frohn, "Refractive-index measurements for the correction of particle sizing methods," Appl. Opt. 30, 4960-4965 (1991).

23. N. Savage, "Laser imaging brings sprays into focus," Laser Focus World 33, 93-97 (1998).

24. A. Tokay and K. V. Beard, "A field study of raindrop oscillations. I. Observation of size spectra and evaluation of oscillation causes," J. Appl. Meteorol. 35, 1671-1687 (1996).

25. H. Lohner, P. Lehmann, and K. Bauckhage, "Detection based on rainbow refractometry of droplet sphericity in liquid-liquid systems," Appl. Opt. 38, 1127-1132 (1999).

26. C. Saekang and P. L. Chu, "Backscattering of light from optical fibers with arbitrary refractive index distributions: uniform approximation approach,” J. Opt. Soc. Am. 68, 1298-1305 (1978).

27. P. Massoli, "Rainbow refractometry applied to radially inhomogeneous spheres: the critical case of evaporating droplets," Appl. Opt. 37, 3227-3235 (1998).

28. L. Kai and P. Massoli, "Scattering of electromagnetic-plane waves by radially inhomogeneous spheres: a finely stratified sphere model," Appl. Opt. 33, 501-511 (1994).

29. L. Kai, P. Massoli, and A. D'Alessio, "Some far-field scattering characteristics of radially inhomogeneous particles," Part. Part. Syst. Charact. 11, 385-390 (1994).

30. Z. S. Wu, L. X. Guo, K. F. Ren, G. Gouesbet, and G. Gréhan, "Improved algorithm for electromagnetic scattering of plane waves and shaped beams by multilayered spheres," Appl. Opt. 36, 5188-5198 (1997).

31. A. A. Kokhanovsky and T. Y. Nakajima, "The dependence of phase functions of large transparent particles on their refractive indexes and shape," J. Phys. D 31, 1329-1335 (1998).

32. G. Gouesbet and G. Grehan, "Generalized Lorenz-Mie theory 
for a sphere with an eccentrically located spherical inclusion," J. Mod. Opt. 47, 821-837 (2000).

33. J. A. Lock, J. M. Jamison, and C.-Y. Lin, "Rainbow scattering by a coated sphere," Appl. Opt. 33, 4677-4690 (1994).

34. D. Marcuse and H. M. Presby, "Optical fiber coating concentricity: measurement and analysis," Appl. Opt. 16, 23832390 (1977).

35. H. M. Presby and D. Marcuse, "Refractive index and diameter determinations of step index glass fibers," Appl. Opt. 13, 28822885 (1974).

36. M. Minnaert, Light and Color in the Outdoors (SpringerVerlag, New York, 1993), pp. 189-198.

37. R. Greenler, Rainbows, Halos and Glories (Cambridge U. Press, Cambridge, UK, 1980), pp. 1-11.

38. J. D. Walker, "Multiple rainbows from single drops of water and other liquids," Am. J. Phys. 44 (5), 421-433 (1976).

39. P. H. Ng, M. Y. Tse, and W. K. Lee, "Observation of high-order rainbows formed by a pendant drop,” J. Opt. Soc. Am. A 15, 2782-2787 (1998).

40. A. L. Aden and M. Kerker, "Scattering of electromagnetic waves from two concentric spheres," J. Appl. Phys. 22, 12421246 (1951).

41. M. Abramowitz and I. A. Stegun, Handbook of Mathematical Functions (Dover, New York, 1970), p. 478.

42. H. C. van de Hulst, Light Scattering by Small Particles (Wiley, New York, 1957), pp. 240-246.

43. C. L. Adler, J. A. Lock, and B. R. Stone, "Rainbow scattering by a cylinder with a nearly elliptical cross section," Appl. Opt. 37, 1540-1550 (1998).

44. C. L. Adler, J. A. Lock, B. R. Stone, and C. J. Garcia, "Highorder interior caustics produced in scattering of a diagonally incident plane wave by a circular cylinder," J. Opt. Soc. Am. A 14, 1305-1315 (1997). 